Archives de sciences sociales des religions

149 | janvier-mars 2010

Varia

\title{
Ascension et déclin du pentecôtisme politique au
} Brésil

\section{Ari Pedro Oro}

\section{OpenEdition}

Journals

Édition électronique

URL : http://journals.openedition.org/assr/21887

DOI : 10.4000/assr.21887

ISSN : $1777-5825$

Éditeur

Éditions de l'EHESS

Édition imprimée

Date de publication : 31 mars 2010

Pagination : 151-168

ISBN : 978-2-7132-2253-5

ISSN : 0335-5985

Référence électronique

Ari Pedro Oro, "Ascension et déclin du pentecôtisme politique au Brésil », Archives de sciences sociales des religions [En ligne], 149 | janvier-mars 2010, mis en ligne le 06 octobre 2010, consulté le 10

décembre 2020. URL : http://journals.openedition.org/assr/21887 ; DOI : https://doi.org/10.4000/assr. 21887

(c) Archives de sciences sociales des religions 


\section{Ari Pedro Oro}

\section{Ascension et déclin du pentecôtisme politique au Brésil}

Au regard des vingt ans de présence des évangéliques (dont les pentecôtistes) dans la vie politique brésilienne - via notamment leur participation à la Chambre des Députés de Brasília - cet article avance l'hypothèse que leur motivation initiale à entrer en politique pour y injecter une forte charge morale devient aujourd'hui un élément contribuant à jeter un certain discrédit sur la politique. On se propose de rendre compte du processus qui a conduit à un tel retournement en présentant les principaux éléments concernant l'histoire et le développement actuel du pentecôtisme brésilien, avant de traiter de la nature de la représentation du « bloc évangélique " dans les sphères de la politique institutionnelle.

\section{Le pentecôtisme au Brésil : quelques données}

L'histoire du pentecôtisme brésilien est généralement présentée en faisant appel à la métaphore des trois vagues. La première couvre la période de 1910 à 1940, au cours de laquelle l'accent est mis sur la glossolalie et le baptême dans le Saint Esprit. Les Églises les plus importantes de cette période sont la Congrégation chrétienne du Brésil, fondée en 1910, à São Paulo, par Luigi Franceson, un Italien venu des États-Unis et l'Église des Assemblées de Dieu, fondée en 1911, à Belém du Para, par les pasteurs suédois Daniel Berg et Gunnar Vingren, également venus des États-Unis. Ce premier pentecôtisme a surtout attiré des travailleurs et des migrants. Sa croissance fut lente.

La deuxième vague s'étend de la décennie des années cinquante jusqu'à celle des années soixante et se caractérise par un accent nouveau mis sur les rituels de guérison divine. Les Églises les plus importantes de cette période sont l'Église de l'Évangile Quadrangulaire, fondée au Canada et entrée au Brésil en 1951 ; Brésil pour le Christ, fondée en 1956 par le brésilien Manuel de Melo, et Dieu est Amour, fondée par David Miranda, en 1962. Ce pentecôtisme reçoit un accueil important auprès des populations venues des zones rurales attirées par 
la croissance industrielle du sud-est brésilien. Il s'agit aussi d'un pentecôtisme qui s'adapte à la religiosité populaire et valorise davantage l'émotion, les symboles et la performance rituelle.

La troisième vague, appelée aussi « néo-pentecôtisme " ${ }^{1}$, s'étend de la décennie soixante-dix à nos jours et met davantage l'accent sur l'exorcisme - basé sur la théologie de la bataille spirituelle -, la théologie de la prospérité, la participation à la politique institutionnelle, l'investissement dans les médias et une certaine libéralisation des mœurs. Les Églises les plus importantes de cette mouvance sont l'Église Universelle du Royaume de Dieu (1977), l'Internationale de la Grâce de Dieu (1980) et l'Église Renaître en Christ (1986). Ce pentecôtisme a gravi l'échelle sociale puisqu'il a attiré aussi des gens des classes moyennes. S'il n'y a pas de frontières claires entre ces trois vagues, elles ne se superposent pas non plus, de sorte que le pentecôtisme brésilien se caractérise surtout par l'interaction de styles empruntés à ces trois vagues (Freston, 1994). Si les pentecôtismes de la première et de la deuxième vague exigeaient de leurs fidèles un certain ascétisme de conduite et un rejet des plaisirs du "monde ", le néo-pentecôtisme autorise les fidèles à participer aux activités du monde, de même qu'il valorise un autre rapport au corps. Désormais, les fidèles sont invités à se vêtir comme ils le désirent et à ne pas mépriser les loisirs et, plus particulièrement, les femmes peuvent faire usage de produits de beauté. Évidemment, cette forme de "libéralisation des mœurs » a des limites. Ainsi, par exemple, l'interdiction des boissons alcoolisées reste de mise, de même que celle de la participation aux fêtes populaires, telles le carnaval.

Selon le dernier recensement officiel de l'an $2000^{2}$, les Églises pentecôtistes brésiliennes les plus importantes par leur nombre de fidèles sont les Assemblées de Dieu, avec 8418154 membres (soit 47,47\% de l'ensemble pentecôtiste), suivies de la Congrégation chrétienne du Brésil (2 489079 fidèles, 14,04 \%), et de l'Église universelle du royaume de Dieu (2 101884 membres, 11,85 \%). Viennent ensuite les Églises de l'Évangile Quadrangulaire (1 318812 membres), Dieu est Amour (774 827 membres) et les autres. Selon la même source, en 2000,

1. Ce terme est néanmoins très controversé et il a déjà provoqué beaucoup de débat. Pour les uns, comme M. Aubrée, ce concept rend compte des changements intervenus dernièrement dans le monde pentecôtiste, au niveau de la doctrine et de l'éthique (1996); pour les autres, comme J.-P. Willaime, la distinction entre pentecôtisme et néo-pentecôtisme reste "floue et contestable ", une distinction « difficilement opératoire " (1999 : 8). Quoi qu'il en soit, comme le remarque André Corten, «le débat sur pentecôtisme et néo-pentecôtisme n’est pas inutile. Il permet de mettre en évidence des transformations générales » (1999 : 176). Sur le débat autour du néo-pentecôtisme en particulier, et des paradoxes du pentecôtisme en général, voir J.-P. Willaime : "Le pentecôtisme : contours et paradoxes d'un protestantisme émotionnel ", Archives de Sciences Sociales des Religions, 105, 1999.

Je remercie André Corten pour l'encouragement à écrire ce texte. Je remercie aussi PierreJoseph Laurent et Sandra Fancello pour leur lecture attentive du manuscrit et leurs suggestions.

2. Cf. l'Institut Brésilien de Géographie et Statistique. 
15,4\% de la population brésilienne se réclamaient d'une appartenance évangélique, dont $67 \%$ du pentecôtisme. Toutefois, une recherche plus récente, réalisée, en 2007, par l'Institut Data Folha de São Paulo, indique que le pourcentage d'évangéliques brésiliens est passé, au total, à $22 \%$. Le tableau suivant donne une vision d'ensemble du champ religieux brésilien. Il établit une comparaison des déclarations d'appartenance depuis le début du $\mathrm{XX}^{\mathrm{e}}$ siècle.

\begin{tabular}{|l|c|c|c|c|c|c|}
\hline \multicolumn{1}{|c|}{ Religions } & 1900 & 1950 & 1980 & 1990 & 2000 & 2007 \\
\hline Catholiques & 98,9 & 93,7 & 89,2 & 82,9 & 73,8 & 64 \\
\hline Évangéliques & 1,0 & 3,4 & 6,6 & 9,0 & 15,4 & 22 \\
\hline Spirites & & & & 1,1 & 1,3 & 2,0 \\
\hline Umbanda/Candomble & & & & 0,4 & 0,3 & 1,7 \\
\hline Autres & & & & 0,06 & 1,2 & 3,0 \\
\hline Sans Religion & 0,1 & 0,5 & 1,6 & 4,7 & 7,3 & 7,0 \\
\hline
\end{tabular}

Tableau I. Cadre comparatif de l'évolution (en \%) des religions au Brésil

La constatation évidente qui ressort de ce tableau est la réduction progressive du nombre de Brésiliens qui se disent catholiques et l'augmentation importante de ceux qui se déclarent évangéliques (parallèlement les "sans religion " passent de $0,1 \%$ à $7 \%$ de la population $)^{3}$. Les confessions évangéliques en progression ne sont pas celles dites historiques (luthériennes, baptistes, presbytériennes et autres) mais bien plutôt celles de la mouvance pentecôtiste et néo-pentecôtiste, dont les fidèles sont désignés par le terme crentes (litt. "croyants »).

\section{L'entrée des crentes dans le champ politique brésilien}

Jusque dans les années quatre-vingts, le pentecôtiste brésilien était fidèle à l'idée que les «croyants » n'avaient pas à se mêler de la politique, celle-ci étant considérée comme relevant du monde séculier. L'activité politique se limitait alors au vote (obligation légale au Brésil). Il existait certes déjà une certaine présence évangélique à la Chambre Fédérale des Députés mais il s'agissait d'une présence insignifiante, composée d'un à trois députés qui se déclaraient membres d’une Église évangélique historique.

3. Parmi les auteurs qui ont essayé de comprendre le retrait catholique et la progression pentecôtiste au Brésil, citons Sanchis, 1994 ; Oro, 1996 ; Fernandes, 1998 ; Mariano, 2001. 


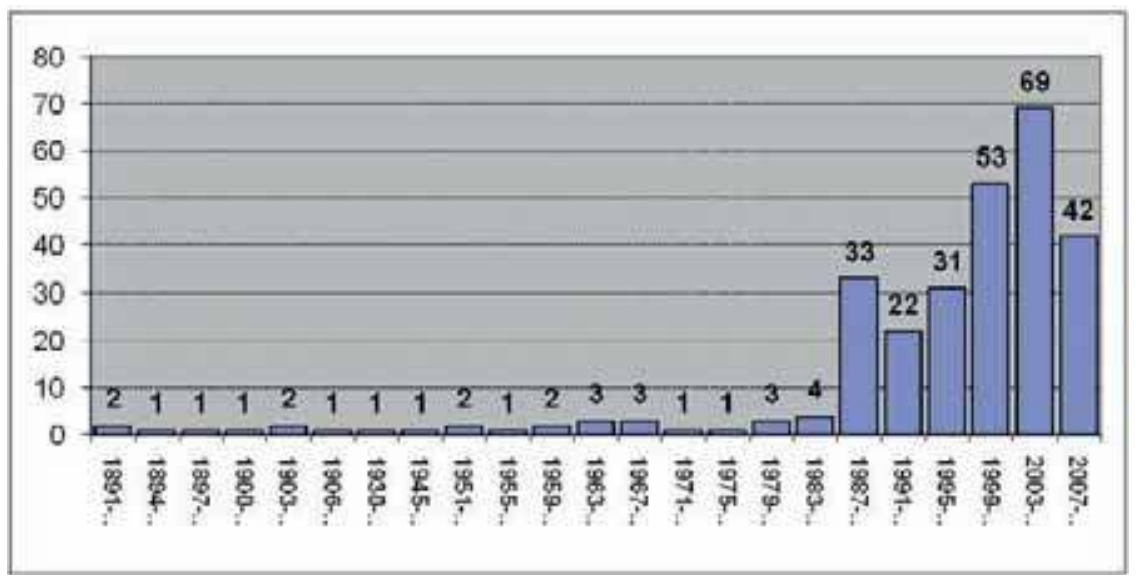

Tableau II. Progression des députés évangéliques à la Chambre Fédérale des Députés de 1891 à 2007

Ce tableau témoigne de la présence évangélique dans la sphère politique institutionnelle dès la fin du XIX ${ }^{\mathrm{e}}$ siècle. La participation évangélique reste très réduite jusqu'à l'élection de la Constituante de 1986 (mise en place en 1987), et qui correspond à la deuxième année du retour de la démocratie au Brésil, après vingt ans de dictature militaire. À ce moment, les évangéliques élisent trente-trois députés. Cette date marque l'entrée du pentecôtisme en politique avec l'élection de dixhuit députés dont quatorze appartenant aux Assemblées de Dieu. En 1990, le «bloc évangélique » à la Chambre des Députés tombe à vingt-deux députés, dont dix-neuf pentecôtistes (six appartenant à l'Église universelle). Aux élections de 1998, le groupe évangélique atteint le chiffre de cinquante-trois députés, dont dix-sept appartenant à l'Église Universelle. En 2002, ce groupe connaît son score le plus élevé à ce jour, avec soixante-neuf députés, dont la majorité est pentecôtiste : vingt-trois proviennent des Assemblées de Dieu et vingt-deux de l'Église universelle. Toutefois, lors des dernières élections (2006), le bloc évangélique connaît une chute considérable, avec seulement quarante-deux députés élus. Sachant que, depuis 1987, la Chambre des Députés à Brasília se compose de cinq cent treize députés, la représentation évangélique a oscillé durant cette période entre 4 et $13 \%$.

Le sociologue et théologien méthodiste, Leonildo Campos, propose une analyse idéal-typique des politiciens issus du milieu évangélique. Jusqu'à la Constituante de 2007, la principale motivation des « politiciens évangéliques » visait à s'opposer à la «soif de pouvoir " de l'Église catholique, laquelle tentait de reprendre quelques-uns des pouvoirs perdus à la suite de la séparation de l'État et de l'Église, survenue en 1889, avec l'avènement de la République. Toutefois, après la Constituante de 1987, entrent en scène les "politiciens du Christ ", issus principalement du milieu pentecôtiste. Cette branche évangélique met en place une action systématique et calculée visant l'espace public et le champ politique. 
Cette nouvelle génération de politiciens soutient l'idéal évangélique qui se caractérise par la mise en place d'une "nouvelle lignée » de politiciens - croyants et honnêtes - qui se nourrissent du « vieux rêve sectaire d'élire un Président de la République évangélique »(Campos, 2006 : 46).

Soulignons que le Brésil n'est pas le seul pays de l'Amérique latine qui connaît une présence évangélique dans la sphère politique. Comme l'avait remarqué J.-P. Bastian en 1997 : « depuis une dizaine d'années la scène politique latinoaméricaine s'est vue investie par une prolifération d'acteurs politiques "évangéliques" et de partis politiques confessionnels, fondés par des clercs ou des laïcs étroitement liés aux nouvelles sociabilités religieuses » (1997 : 97). Bastian parle à ce propos d'une "confessionalisation » de la politique.

Vingt ans après la formation du premier «bloc évangélique » à la Chambre des Députés de Brasilia, on dispose de différentes études en sciences sociales sur le sujet qui permettent d'éclairer non seulement les motivations de l'insertion pentecôtiste dans le champ politique mais également les pratiques, et les raisons de la fluctuation du nombre de leurs élus. La synthèse de ces recherches présentée ci-dessous porte sur le pentecôtisme en général, sachant qu'il existe des variations selon les différentes Églises.

\section{Tradition millénariste et guerre spirituelle}

L'entrée du pentecôtisme en politique peut être attribuée à deux motivations importantes, l'une d'ordre symbolique, à laquelle les fidèles sont très sensibles, et l'autre d'ordre pratique, plutôt restreinte aux groupes dirigeants des Églises.

L'une des motivations, qui a vraiment mobilisé les fidèles pentecôtistes en politique, au point de renverser la conception traditionnelle selon laquelle «le croyant ne se mêle pas de politique ", découle à la fois du principe millénariste et de la notion de "guerre spirituelle". Ce changement résulte le plus souvent d'une adaptation de ces deux conceptions, dont l'intensité varie selon les Églises. La composante millénariste - importante dans la culture religieuse brésilienne mise en avant par certaines Églises pentecôtistes et néo-pentecôtistes, à des degrés divers, vise l'émergence d'un nouveau monde, d'une nouvelle société, porteuse d'une nouvelle morale publique et d'une nouvelle éthique en politique, une nation guidée par Dieu, obéissant à la formule biblique : "Heureux le peuple dont Yahvé est le Dieu, la nation qu'il s'est choisie en héritage !» (Psaumes, 12). Pour y parvenir, il faut passer par la " guerre spirituelle » contre les forces invisibles du Mal qui agissent dans le monde et qui sont également à l'œuvre dans la sphère politique ${ }^{4}$. Selon cette conception, les forces du Mal sont à l'origine de

4. La croyance aux forces démoniaques est partagée par $89 \%$ des évangéliques brésiliens (Fernandes, 1996 : 81). Elle baisse à $44 \%$ parmi la population brésilienne en général (Revista Veja, 2-04-1997). En Europe, en 1990, cette croyance était partagée par 23 \% des citoyens de 
la corruption et des attitudes amorales des politiciens. C'est pourquoi l'évêque et député Rodrigues, de l'Église Universelle du Royaume de Dieu ${ }^{5}$, déclarait en 2002 : «mon engagement en tant que député vise à maintenir l'éthique. Je veux combattre la corruption ${ }^{6}$. Une année auparavant, ce même leader politique pentecôtiste affirmait : " les esprits qui agissent dans la politique sont les esprits dominateurs, les princes des ténèbres ${ }^{7}$. Dans ce combat spirituel, le pouvoir pentecôtiste mise sur "la puissance du Saint Esprit ", le "pouvoir de Notre Seigneur Jésus ", capable de libérer la politique des puissances démoniaques.

Cette approche spirituelle du politique, mise en avant par les pasteurs et les prédicateurs pentecôtistes, n'est pas sans rapport avec la mentalité cosmologique et la sensibilité enchantée, voire "magique ", du pentecôtisme en particulier et des cultures religieuses latino-américaines en général. La sécularisation des institutions politiques et de l'État ne semble pas être suivie par la sécularisation des idéologies politiques, en raison d'une conception qui envisage le rapport entre le public et le privé d'une façon différente de celle de la tradition libérale (Seman, 2000).

Une autre déclaration de l'évêque et député Rodrigues, en l'an 2000, synthétise cette vision pentecôtiste millénariste en politique : "J'en appelle au peuple de Dieu à ce que nous nous rassemblions autour de nos idéaux et que nous n'ayons pas peur de participer au processus politique, tout en élisant des hommes et des femmes qui croient au Seigneur Jésus pour que notre société soit transformée et puisse être orientée selon les vraies principes chrétiens " ${ }^{8}$. Le sociologue Paul Freston a recueilli le propos d'un pasteur des Assemblées de Dieu qui va dans le même sens : «La transformation du Brésil doit commencer par la restauration spirituelle de la nation moyennant la présence des hommes de Dieu aux postes de commandants » (Freston, 1997). Le théologien méthodiste, Leonildo Campos, souligne que le rêve d'un nouveau début social et d'une reconstruction sociopolitique prévaut dans l'ensemble du protestantisme brésilien et il s'exprime par

quinze pays, divisés entre pays catholiques, pluriconfessionnels et luthériens (Pace, 1998). Ces dernières années, la croyance dans le démon progresse aussi bien au Sud qu'au Nord. Pour D. Hervieu-Léger, l' "augmentation de la croyance au diable dans la France contemporaine " (1999 : 49) s'inscrit dans une modernité religieuse marquée par les frustrations psychologiques et sociales exacerbées par la crise économique, la disqualification du politique et l'absence de perspectives (id., 1999: 51). La sociologue fait l'hypothèse d'un rapport entre le « retour du diable » et les diverses modalités de diabolisation de l'autre (l'étranger, l'Arabe ou le juif, mais aussi les technocrates, les politiciens) (id., $1999: 52)$.

5. Cet évêque a été élu deux fois député fédéral par les fidèles de son Église de l'État de Rio de Janeiro, en 1998 et en 2002. Il a aussi fondé l'Église universelle en Argentine et en Espagne et il a travaillé en tant que pasteur au Portugal, en Angola, en Afrique du Sud et au Mozambique avant de se consacrer totalement, en 1996, à la tache de coordinateur politique national de l'Église universelle. On lui attribue le succès politique de cette Église au fil des années.

6. Jornal Folha Universal, 19 octobre 2002.

7. Jornal do Brasil, 29 octobre 2001.

8. Jornal Folha Universal, 6 août 2000. 
ces mots d'ordre : "Le Brésil sera un autre pays quand il aura à sa tête un homme de Dieu» (2000 : 21). La déclaration d'un membre de l'Église universelle, Dilmair dos Santos, illustre cette approche à la fois millénariste et belliqueuse : "Nous, de l'Église Universelle, nous avons une proposition radicale pour transformer la société et pour construire une nouvelle et grande nation. Il s'agit d'une proposition radicale étant donné que l'Église prêche contre les vices, les drogues, l'adultère, la corruption. Tout cela est l'œuvre du démon, du dévorateur, qu'on doit combattre ${ }^{9}$.

La seconde motivation au fondement de l'investissement du pentecôtisme dans la sphère politique est plus restreinte aux dirigeants des Églises et à leur clergé. Il s'agit d'une raison d'ordre pratique, à savoir, d'une part, soutenir dans le parlement les intérêts des Églises ${ }^{10}$ et, d'autre part, établir des liens avec les pouvoirs publics afin d'obtenir des bénéfices de l'État, tels que des appuis à des programmes et projets sociaux, mais aussi des concessions de radio-émetteurs et de télévision. Historiquement, ces bénéfices de l'État étaient drainés vers l'Église catholique. Le pasteur A. Reis, des Assemblées de Dieu de Porto Alegre, déclarait en 2004 : "Pourquoi seule l'Église catholique a-t-elle le droit de recevoir l'aide de l'État? Nous savons aujourd'hui que nous pouvons nous aussi bénéficier de l'aide du gouvernement. Pour cela il faut avoir des députés de notre Église ». Ces positions des évangéliques témoignent de leur compréhension de l'importance prise par la politique dans la conduite journalière de la vie sociale. À ce propos, le pasteur et député de l'État du Rio Grande do Sul, Sergio Peres, de l’Église Universelle, explique : «De nos jours, tout passe par la politique. Même le pain qu'on mange aujourd'hui, la farine, le blé, ce sont des décisions politiques. C'est pourquoi l'Église a besoin de quelqu'un à l'intérieur de la politique ${ }^{11}$.

\section{L'engagement et la pratique des politiciens évangéliques}

Le Brésil est un pays laïque depuis la proclamation de la République survenue en 1889. Avant cette date, c'est-à-dire pendant toute la période coloniale et impériale, de 1500 à 1889, le catholicisme était la religion officielle de l'État brésilien. Toutefois, la première Constitution républicaine, en 1891 établit le principe de la séparation de l'Église et de l'État, garantit la liberté religieuse à chaque individu, le libre exercice du culte à tous les groupes religieux, ainsi que le traitement isonomique de l'État envers toutes les religions. Mais, dans la pratique, l’Église catholique n'a cessé de jouer un rôle déterminant dans la définition

9. Propos recueilli par V. Pedde (2005).

10. Le pasteur et député fédéral de l'Église universelle, Paulo Gouvea, explique dans un entretien accordé en 2006, que : "l'Église doit avoir des députés pour résoudre certains problèmes ». De même, le conseiller municipal de Porto Alegre, pasteur de l'Universelle, Almerindo Filho, ajoute que : "Le but principal pour qu'un pasteur quitte l'autel et entre dans la politique est la défense de l'Église ».

11. Propos recueilli par V. Pedde (2005). 
des rapports État et religion au Brésil. Il s'agissait de poursuivre la catholicisation de la société et le maintien de ses privilèges, aux niveaux social, éducationnel et patrimonial. Pour l'État, les bons rapports avec l'Église catholique lui assurait une meilleure légitimation sociale dans certains moments historiques (Löwy, 1997). Dès lors, jouant sur son importance symbolique et politique acquise au long de trois siècles, l'Église catholique réussit à introduire dans la Constitution de 1934 la possibilité de «collaboration » entre État et religions. Ce dispositif sera maintenu dans les Constitutions suivantes dont la dernière, de 1988. Autrement dit, le principe de séparation est nuancé et l'Église catholique en tire des bénéfices symboliques, politiques et économiques.

Signalons, à ce propos, la vive polémique déclenchée par le "Concordat " signé entre le Brésil et le Vatican, en 2008. Ce texte est actuellement au Sénat, après avoir été approuvé par la Chambre des Députés à Brasilia. Ce concordat, composé de dix-neuf articles, reconnaît le statut juridique de l'Église catholique et traite de questions cruciales liées au devenir du catholicisme au Brésil, telles que l'enseignement religieux dans les écoles publiques, les écoles catholiques, l'assistance religieuse dans les prisons et les hôpitaux, les séminaires et la reconnaissance des diplômes et les bénéfices fiscaux. Plusieurs organisations et associations nationales se sont opposées à ce concordat qui met en cause le principe de la laïcité inscrite dans la Constitution Nationale. Au Congrès National, la réaction la plus forte a été orchestrée par le "bloc évangélique " qui trouve dans le privilège accordé à l'Église catholique de quoi donner raison au Pasteur Reis et à bien d'autres évangéliques quant à leur investissement dans les espaces politiques pour défendre leurs intérêts. En un mot, au Brésil la «séparation » Église et État ou religion et politique «ne s'est jamais produite historiquement » (Giumbelli, $2002: 50)$.

Concernant la pratique du «bloc évangélique » à la Chambre des Députés, on peut observer en premier lieu que ce bloc n'est pas porteur d'une cohésion politique, sauf à propos de certains sujets à caractère moral, tels que l'avortement et l'union civile des homosexuels. Comme le souligne Alexandre Brasil Fonseca: « une analyse du comportement du congressiste évangélique montre que le bloc évangélique est un mythe » (Fonseca, 2009). Il ne faudrait pas pour autant sousestimer le pouvoir politique des évangéliques à la Chambre des Députés. Représentant aujourd'hui près de $8 \%$ du parlement à Brasilia, ils forment un groupe de pression équivalent aux partis de taille moyenne et particulièrement efficace aussi bien pour faire obstacle à certains projets d'ordre moral (comptant pour cela sur l'appui des catholiques conservateurs) ${ }^{12}$, que pour modifier des projets déjà approuvés, tels le Code Civil ${ }^{13}$.

12. Les deux projets de lois qui traînent dans le parlement brésilien sont celui de la légalisation de l'avortement et celui du mariage civil des homosexuels.

13. Il s'agit de la mobilisation qui a abouti au retrait de ce code de l'exigence du paiement d'impôts de la part des Églises. 
En même temps, il s'agit d'un groupe de députés répartis dans la plupart des partis politiques qu'ils soient de droite, du centre ou de gauche, ce qui conduit ses membres à voter dans des directions différentes et empêche de le considérer comme un bloc politiquement conservateur. En réalité, comme le remarque André Corten : "le pentecôtisme n'est ni plus ni moins conservateur que l'Église catholique " (1997: 18). On ne voit donc pas émerger un "mode d'être évangélique ou pentecôtiste en politique »(Machado, 2006). Les députés évangéliques, y compris les pentecôtistes, semblent reproduire la culture politique brésilienne qui place les élus dans l'obligation de donner suite aux demandes de leurs « bases électorales » (Bezerra, 1999), leurs communautés d'attachement, afin de maintenir leur prestige (Kurschnir, 1996). Les politiciens évangéliques agissent comme tous les politiques, pour la défense des intérêts de leurs Églises et pour relayer les demandes de leurs fidèles en adoptant des positions clientélistes dont les effets pratiques varient selon les appartenances et les opportunités ${ }^{14}$. Ainsi, M. Santos, qui a étudié la pratique politique des pasteurs-conseillers municipaux de Porto Alegre, observe que ces derniers " cherchent à obtenir des bénéfices pour leur Église [l'Universelle], de même qu'ils tentent de satisfaire les demandes de leurs "bases électorales" (2005: 115). La chef de bureau du pasteur et conseiller municipal, Valdir Caetano, déclarait à ce chercheur : " le travail des députés et des conseilleurs municipaux est très lié à leurs bases, n'est-ce pas ? Notre base d'appui est notre Église. C'est pour elle qu'on doit travailler» (id., 2005 : 158). À quelques exceptions près, ces politiciens n'agissent donc pas au nom de l'intérêt religieux en général, pas même celui de l'intérêt commun des "évangéliques ", mais plutôt au nom de leurs propres dénominations. Les politiciens pentecôtistes, selon le sociologue R. Mariano, "se comportent comme des émissaires de leurs Églises»(2001: 7).

Cette pratique politique reproduit les rapports clientélistes qui, selon Murilo de Carvalho (1997), traversent l'histoire politique du Brésil. Concrètement, les politiciens essaient d'obtenir les votes des électeurs en échange de bénéfices publics,

14. Les demandes peuvent être de toutes sortes mais les plus récurrentes seraient la quête d'argent, de travail, d'assistance juridique, d'assistance médicale, de régularisation des documents personnels. À la Chambre Municipale de Porto Alegre, Claudio, le responsable du bureau du conseiller de la Mairie, Almerindo Filho, pasteur de l'Universelle, déclarait que la demande principale concerne l'argent: «dans la première année du mandat (du pasteur et conseiller municipal) nous donnions beaucoup d'argent ici (...). Les personnes demandaient et nous donnions. Mais à partir de la deuxième année nous avons changé : nous avons étudié ce que la Mairie peut apporter comme aide, ce que l'État peut faire, et alors nous acheminions les gens vers les bons endroits. Mais les gens pensent que nous avons toujours de l'argent. Surtout à cette époque de l'année, le mois de décembre est terrible. Les gens viennent de tous les coins de Porto Alegre " (Santos, 2005). La responsable du bureau de l'autre conseiller de la Mairie, Valdir Caetano, lui aussi pasteur de l'Universelle, affirmait : "On prend de l'argent de notre propre poche. Le mois dernier j'ai donné tous mes tickets de transport... ». Elle aussi disait cependant que le bureau réorientait vers les endroits susceptibles d'embaucher les gens et de les aider. Tous les deux soulignèrent aussi une autre requête souvent formulée par les gens qui s'adressent aux bureaux des conseillers municipaux : l'aide et les conseils spirituels (id., 2005). 
tels qu'emplois, arrangements fiscaux, services sociaux (surtout en matière de santé), qu'ils obtiennent grâce à leur capacité à influencer les gouvernements. S'installe ainsi un rapport de complémentarité entre clientélisme et populisme (Diniz, 1982) et entre clientélisme et corporatisme (Kaufman, 1977). Selon J.-P. Bastian, le corporatisme et le clientélisme caractérisent autant le pentecôtisme que la culture politique latino-américaine. C'est l'affinité élective qui explique « l'irruption politique du religieux sectaire " et dans les deux cas, "les styles d'autorité et les mécanismes de domination y sont marqués par la récurrence de l'autoritarisme » (1997: 105).

Enfin, en analysant le comportement politique et les discours des politiciens pentecôtistes brésiliens, surtout ceux de l'Église universelle, on observe une sorte de sécularisation de leurs engagements : ils débutent en tant qu'évêques ou pasteurs mais deviennent, avec le temps, des politiciens comme les autres, laissant de côté le langage religieux et leurs intentions de moraliser la société. Au début de leur mandat, le parlement est conçu comme le prolongement de la chaire du prédicateur. Lors de son premier discours politique, le pasteur de l'Universelle, Almerindo Filho, élu conseiller municipal de Porto Alegre déclare : "C'est la première fois que je monte officiellement à la tribune. Je compare cet endroit à un autel de l'Église ». Son but au parlement, ajoute-t-il, " est la diffusion de la parole de Dieu ". Mais à mesure que le temps passe, on voit que les députés et les conseillers municipaux font de moins en moins référence à la Bible et commencent à prononcer des discours semblables à ceux des autres acteurs politiques. Au fil de leurs mandats, comme le dit Santos (2005), ils passent « de pasteurs à conseilleurs municipaux ", ou " de pasteurs à députés ". Et cela s'observe dans tous les cas, jusqu'à la dérive vers une pratique politique dépourvue d'éthique, comme on le verra par la suite. De même, au fur et à mesure de leur mandat, leurs prises de positions suivent de plus près les orientations des partis, au détriment de celles des Églises. Le conseiller municipal de Porto Alegre, Valdir Caetano, pasteur de l'Universelle, a commencé par déclarer : "Pour moi, ce qui compte le plus c'est l'Église. D'abord l'Église et puis la politique. Dans mon cœur, je suis d'abord pasteur et après politique ". Cependant, dans la pratique, la fidélité à l'Église semble être peu à peu remplacée par l'allégeance aux partis politiques. Les députés pasteurs sont obligés de s'adapter aux lois en vigueur, d'agir dans les limites constitutionnelles et de suivre les normes établies pour le fonctionnement des institutions politiques et le règlement des Chambres législatives. Si ce fait révèle la capacité d'intégration des religieux politiciens à la politique institutionnelle, il montre également l'importance institutionnelle du politique, auquel les Églises et les politiciens religieux doivent se soumettre ${ }^{15}$.

15. Le philosophe brésilien Renato Janine Ribeiro avance que ce pouvoir d'attraction du politique constitue une force "vampiresque " censée neutraliser ce qu'il y a de mieux dans la société civile : le politique devient un "cimetière de ce qu'il y a de mieux dans la société " (2002 : 104-105). 
Les dirigeants des Églises, surtout de l'Universelle, mais aussi des Assemblées de Dieu et de la Quadrangulaire, se montrent finalement peu satisfaits de la prestation de leurs politiciens au parlement. Après un ou deux mandats, ils remplacent les pasteurs ou les évêques devenus politiciens par d'autres membres du clergé dans l'espoir que les nouveaux élus resteront fidèles aux Églises. Ajoutons que le retrait de l'appui institutionnel accordé aux politiciens installés est dû, aussi, au fait qu'un certain nombre d'entre eux se sont compromis dans des affaires de corruption ${ }^{16}$.

\section{Les promesses non tenues des hommes de Dieu}

Depuis quelques années, on constate une démobilisation de l'engagement des pentecôtistes en politique : constat qui concerne toutes les Églises, à commencer par l'Église universelle, qui a pourtant connu un succès politique croissant depuis $1990^{17}$. L'échec politique de cette Église a commencé lors des élections municipales de 2004, quand elle n'a fait élire que soixante-dix conseillers municipaux dans tout le pays alors qu'en 2000, elle en avait faire élire trois cent soixante. L'explication en interne de cet échec, donnée par l'évêque Paulo Moreira, de Porto Alegre, mettait en cause le « remplacement des pasteurs par des “ouvriers" de l'Église en tant que candidats ". Mais même si tel était vraiment le cas, cela n'explique pas l'échec électoral, surtout si l'on tient compte de la force mobilisatrice qui existait jusqu'à ces dernières années dans cette Église "charismatique, centralisée et non participative " (Carneiro, 1998; Oro, 2003), qui parvenait à faire voter jusqu'à $95 \%$ de ses fidèles selon les indications du groupe dirigeant de l'Église (Fernandes, 1996) ${ }^{18}$. C'est donc ailleurs que l'on doit chercher l'explication de l'échec politique de cette Église néo-pentecôtiste, en particulier, et plus globalement des autres. Le « désenchantement » du politique s'est produit surtout au niveau des fidèles, et il s'agit d'un double désenchantement.

Tout d'abord, après plus de vingt ans d'investissement des Églises dans la sphère politique, celle-ci demeure telle qu'elle était auparavant : privée de valeurs éthiques et morales, et dont les représentants ne contribuent pas, finalement, à

16. Dans plusieurs États brésiliens, l'Église universelle, plus que toute autre, a dû faire face à un problème inattendu, à savoir que la plupart des politiciens élus avec l'appui de l'Église, aussi bien des conseillers municipaux que des députés, mais remplacés lors des nouvelles élections par d'autres noms, se sont représentés à leur propre initiative, en mettant en avant leur qualification religieuse dans leurs publicités. Le résultat, néanmoins, est qu'en l'absence d'appui institutionnel, ils ont subi une éclatante défaite.

17. Signalons que cette Église est présente de nos jours dans cent vingt pays mais que c'est seulement au Brésil qu'elle a investi le champ politique. Elle a fait quelques essais, sans succès, au Portugal et en Argentine (Oro, 2003). En Afrique, affirme A. Mary, notamment en Côted'Ivoire, "le souci de s'insérer dans l'espace politique national (...) n'est pas une priorité de l'EURD... » (2002: 474).

18. Il fut un temps, où l'expression "vote fidèle ", désignait de façon ironique l'idée que l'Église universelle pouvait faire élire un poteau, si elle le voulait. 
la moralisation du champ politique. La corruption est toujours là, la politique n'est pas délivrée de ses maux.

Quelques propos recueillis dans le milieu pentecôtiste, à Porto Alegre, témoignent du sentiment des fidèles à ce sujet : "La politique fut, est et sera toujours pourrie "; "On ne peut rien faire pour changer la politique, parce que la politique est le royaume du Satan. C'est comme ça depuis le début du monde. C'est pourquoi nous ne devons pas participer à la politique. Au contraire, nous devons nous en tenir loin »; "Pour moi politique et péché c'est la même chose "; "Politique et corruption vont ensemble dans ce pays »; "Tu vois les nouvelles à la radio, à la télé ? C'est toujours la violence et la corruption dans la politique »; «La politique est une chose du Diable »; «On ne doit pas mélanger la religion et la politique ».

De fait, dans les quelques dernières années, la presse a révélé plusieurs cas de détournement de fonds et d'appropriation privée d'argent public par les politiciens de tous bords, à Brasilia comme ailleurs. Certains de ces faits ont débouché sur la formation de quatre Commissions parlementaires mixtes d'investigation au Congrès National (CPMI) : celle de la poste (mai 2005), celle des bingos (juin 2005), celle du mensalão (juillet 2005) et celle des sanguessugas (août 2006) ${ }^{19}$.

Un désenchantement encore plus grand a atteint les fidèles qui voient que nombre de députés issus du milieu évangélique sont impliqués dans des scandales de corruption. Un membre de l'Église Quadrangulaire de Porto Alegre s'interroge : "Où sont-ils ces hommes de Dieu qui ont assumé un "compromis éthique avec Dieu” ? (...) Au lieu d'influencer positivement la politique, malheureusement c'est la politique qui les a influencés, d'une façon très négative ". À son tour, le pasteur A. Reis, des Assemblées de Dieu de Porto Alegre, ajoute : "Les politiciens évangéliques se laissent corrompre ; ils sont vaincus par l'argent. C'est le pouvoir de Satan. Ces quatre, cinq ou dix députés évangéliques ne vont pas pouvoir changer la politique. Nous devons être toujours attentifs. Mais la politique n'est pas l'endroit des hommes de Dieu. Tous [les pentecôtistes] ceux qui se mettent en politique tôt ou tard, ils scandalisent l'Église (AD). C'est pourquoi aujourd'hui même le peuple évangélique ne fait plus confiance aux politiciens évangéliques ».

La pasteure J. N., de l'Église Maanaim, est, pour sa part, encore plus critique envers les politiciens évangéliques : "Ils sont tous corrompus. C’est un scandale.

19. La CPMI de la Poste a été créée pour analyser les dénonciations de corruption dans les entreprises de l'État, surtout dans la Poste. La CPMI des bingos avait pour but d'enquêter sur une négociation entamée entre une entreprise de technologie et une banque de l'État, la Caixa Econômica Federal, ou encore l'implication des maisons de jeu dans le crime organisé. La CPI du Mensalâo avait pour but d'enquêter sur les bénéfices pécuniaires attribués tous les mois aux députés par le gouvernement afin qu'ils soutiennent certains projets d'intérêt du Pouvoir Exécutif. La CPI des Sanguessugas devait enquêter sur l'acquisition d'ambulances pour les municipalités et les bénéfices financiers attribués à certains députés. 
C'est une honte pour nous ". Mais elle semble par ailleurs accepter et même justifier cette conduite des « hommes de Dieu » qui s'engagent dans la politique en soulignant que cela n'est pas nouveau et qu'on la retrouve même dans l'Ancien Testament : "Regardez dans le Livre des Juges. On voit là que les hommes que Dieu Lui-même a choisi pour conduire le peuple, pour être à la tête du peuple, pour combattre le Mal, pour être leurs représentants, ils ont été eux aussi corrompus. Regardez ce qui s'est passé avec Gédéon, Samson, Samuel et même avec Salomon? Tous ont été corrompus ; ils ont tous nié Dieu».

Les affirmations de ces deux pasteurs rejoignent le constat du sociologue brésilien Ricardo Mariano selon lequel, malgré les discours moralisateurs des campagnes électorales, " plusieurs parlementaires pentecôtistes, membres d'Églises et de partis conservateurs ont été la source de plusieurs scandales liés à des détournements de fonds publics et se sont révélés d'une avidité mercantile qui ne les différentie en rien de celle des pires de leurs collègues non croyants " (2001 : 13-14). On se souvient des mots de l'évêque Rodrigues, le plus important coordinateur politique de l'Église universelle, élu deux fois député fédéral, qui déclarait : "Je veux combattre la corruption ». Il fut pourtant accusé, lui aussi, de plusieurs scandales et, pour éviter son expulsion de la Chambre des Députés, il renonça à son mandat en septembre 2005. Une année auparavant, le Conseil des Évêques de l'Universelle l'avait déjà démis de ses fonctions d'évêque et de coordinateur politique de l'Église. De même, au second semestre 2006, quand furent annoncés les noms des députés impliqués dans le "Scandale des Sanguessugas» - concernant l'acquisition d'ambulances pour les municipalités - on comptait trente et un évangéliques parmi les soixante-douze accusés, c'est-àdire presque la moitié du "bloc évangélique ", composé alors de soixante-neuf députés. L'EURD a été l'Église la plus atteinte puisque seize de ses députés ont été accusés, suivie par les Assemblées de Dieu avec dix députés, l'Église baptiste avec deux députés, l'Église Quadrangulaire avec deux députés et l'Internationale de la Grâce de Dieu avec un député. Sur trente et un accusés, vingt-sept ont été condamnés.

L'impact de ces scandales sur les électeurs évangéliques est très grand : lors des élections d'octobre 2006, l'EURD n'a fait élire que cinq députés au lieu des vingt-deux élus quatre ans auparavant; les Assemblées de Dieu n'ont eu que douze élus au lieu de vingt-trois en 2002. Selon les observateurs, «l'ennui, la frustration et la révolte subis par les électeurs évangéliques les a conduits à une vengeance attendue dans les urnes. C'est ce qui s'est passé (...) 2006 fut l'année de la débâcle évangélique (en politique) » (Mariano, Hoff, Toty, 2006 : 70-73). À cette déception, il faut ajouter le fait que les fidèles considèrent que les pasteurs élus députés sont plutôt parmi les meilleurs cadres ecclésiastiques et que les Églises sont privées de leurs compétences lorsqu'ils sont élus. Parallèlement, les pasteurs, une fois reconvertis en politiciens, fournissent une prestation politique insignifiante, plusieurs d'entre eux demeurant méconnus de la société en général pendant des années et même inexistants à l'intérieur du monde politique, à Brasilia ou 
ailleurs. Ils font partie du "bas clergé » à la Chambre des Députés. Aucun d'entre eux n'a occupé un poste important, aucun n'est devenu leader d'un parti politique, ni n’a gagné en rayonnement en tant que député. Le pasteur A. Reis rappelle que «notre actuel député fédéral [des Assemblées de Dieu] était notre leader principal, un sage, un vrai homme de Dieu. Mais depuis qu'il a été élu, il est toujours à Brasilia, on le voit très rarement ici [à Porto Alegre]. Mais je ne l'ai jamais vu à la télé. Personne ne le connaît. Dommage. On a perdu un grand pasteur qui est devenu un député médiocre " ${ }^{20}$. Comme le souligne Mariano, la plupart des députés évangéliques n'apparaissent dans les médias que lorsqu'ils sont impliqués dans des scandales de corruption.

Rappelons que l'un des thèmes discursifs de mobilisation des électeurs, à l'intérieur des Églises pentecôtistes, tournait justement autour de la moralisation de la politique. Voir que la corruption se maintient et, pire encore, que leurs propres pasteurs désignés comme "les hommes courageux du Seigneur ", "les hommes de Dieu ", " les soldats de Jésus Christ ", y sont mêlés, a été pour nombre de croyants un coup dur et source d'une grande déception. Ainsi, le sujet de la corruption et du déficit de la morale qui, au début, a attiré les pentecôtistes vers la politique en promettant sa purification, devient aujourd'hui la source de leur éloignement et du renforcement de l'idée selon laquelle religion et politique ne doivent pas s'entremêler. La sphère politique appartient au "monde ", elle est, par définition, placée sous l'emprise du démon et demeure un espace dangereux, un lieu de tentation qui peut faire tomber même les " courageux du Seigneur " 21 . Il faut ajouter que les pentecôtistes ne sont pas seuls à subir ce désenchantement vis-à-vis de la politique au Brésil. Selon Renato Janine Ribeiro (2002), le discrédit est plus ou moins général dans ce pays ${ }^{22}$.

\section{Conclusion : rebondissement ou accommodement}

On ne peut sous-estimer la capacité d'adaptation du pentecôtisme aux nouvelles situations ni son aptitude à construire de nouveaux discours susceptibles d'ouvrir d'autres chemins et à trouver de nouvelles motivations pour se maintenir

20. Nous ne sommes pas encore en mesure d'évaluer les changements que cette constatation, énoncée par le pasteur Reis, produira sur les stratégies de captation du pouvoir politique par des Églises comme les Assemblées de Dieu. Il faudra pour cela attendre les prochaines élections qui auront lieu le 3 octobre 2010.

21. Les propos recueillis dans le milieu pentecôtiste se multiplient dans ce sens : "la politique est menée par le démon »; "la politique n’appartient pas au peuple de Dieu ».

22. Selon cet auteur, "l'opinion publique brésilienne est très sceptique par rapport aux politiciens " (Ribeiro, 2002 : 99), étant donné que leur prestation est en grande mesure associée au patrimonialisme, au népotisme et à la corruption. D’après l'éditorial du journal Folha de São Paulo du 31 juillet 2005, intitulé justement « désenchantement politique », $49 \%$ de la population brésilienne ne croient pas à l'existence de politiciens honnêtes. Il souligne aussi que dans certains pays de l'Amérique latine, l'expression "que se vayan todos " marque la forte méfiance vis-à-vis des politiciens. 
dans la sphère politique. Malgré le discrédit qui a atteint les pentecôtistes brésiliens, ces dernières années, deux préoccupations se dessinent permettant d'envisager un éventuel rebondissement en politique ou des formes d'accommodement.

Tout d'abord, on constate qu'une partie des électeurs pentecôtistes considèrent désormais que l'idée de " purification de la politique » reste de l'ordre du discours et de l'utopie, impensable en pratique. Après avoir vécu et expérimenté les déceptions et les échecs par rapport aux changements attendus de l'action politique, ces électeurs considèrent qu'il faut distinguer désormais entre ce que les pasteurs "disent vouloir, (...) ce qu'ils sont et ce qu'ils font en réalité », comme le dit, à sa manière, Marcel Gauchet à propos des fondamentalismes ou des intégrismes religieux (2003: 53).

Par ailleurs, si le ressort symbolique qui justifiait l'entrée des pentecôtistes en politique n'a plus d'efficacité, ou du moins, la force mobilisatrice qu'elle avait auparavant, la raison " pratique » commence à reprendre le dessus. On observe, en effet, une mobilisation du discours utilitariste visant à légitimer l'engagement politique. Ce revirement se fonde désormais sur une stratégie de captation des ressources nécessaires à la survie de ces Églises minoritaires dans une situation dominée par l'Église catholique et marquée par la compétition avec les autres Églises pentecôtistes ou évangéliques. C'est ce discours que l'on retrouve en particulier chez les cadres dirigeants des Églises et qui est de plus en plus diffusé auprès des fidèles de certaines Églises pentecôtistes. Telle est l'opinion de la pasteure J. N., de l'Église Maanaim, de Porto Alegre : "On doit malgré tout s'engager dans la politique si l'on veut avoir quelque chose. Tout passe par la politique. Autrement le gouvernement draine l'argent et les moyens seulement vers l'Église catholique. En outre, si l'on n'a pas de représentants dans la politique, ils [les catholiques] risquent de voter des lois qui nous obligent à fermer nos portes. On doit donc être dans la politique pour voir de près et surveiller ce que les autres sont en train de faire et surtout empêcher qu'ils votent des lois qui nous portent des préjudices ». "Quoi qu'il en soit, ajoute l'ex-président des Assemblées de Dieu de Porto Alegre, Joao Ferreira Filho, l'Église (AD) doit lutter pour maintenir au Parlement une voix qui la représente et la soutient ». À ce propos, un fidèle de l'Universelle synthétise la pensée de beaucoup d'autres : "Il faut avoir des pasteurs de notre Église [Universelle] dans la politique si on veut continuer à garder les portes de nos églises ouvertes »(Pedde, 2005).

Un exemple de l'efficacité actuelle des politiciens évangéliques au Congrès National est fourni par l'affaire du concordat entre l'Église catholique et l'État brésilien. Pour les évangéliques, ce document cache l'intention de redonner à l'Église catholique son statut de religion officielle du pays. Ils se sont d'abord opposés fermement à l'approbation du document, avant de se raviser dans un second temps, face à la pression du gouvernement, et de parvenir à faire adopter une «Loi générale des religions » ou "Loi des cultes », proposée par le député George Hilton de l'Église Universelle. Le nouveau texte est une réplique du 
concordat entre le Vatican et l'État (avec les mêmes dix-neuf articles), mais le mot catholique se trouve simplement remplacé par l'expression «toutes les confessions religieuses ». Les deux lois ont été votées, le 24 août, par la Chambre des Députés et devraient l'être prochainement au Sénat.

Ainsi, malgré la réduction de la représentation du «bloc évangélique » au Congrès National, ce groupe religieux conserve un poids politique important puisqu'il est capable de contraindre l'État à dialoguer avec lui, et donc de mettre un terme au monopole d'interlocuteur religieux unique du catholicisme. Cependant, comme le note J.-P. Bastian, ce fait n'est pas nouveau au Brésil et il est même récurrent dans d'autres pays d'Amérique latine. Il contribue à modifier les conceptions de la laïcité, ainsi que l'évolution des rapports entre l'État et la religion dans cette région du monde (1997) ${ }^{23}$.

Ari Pedro ORO Porto Alegre - Université Fédérale du Rio Grande do Sul, arioro@uol.com.br

\section{Bibliographie}

BASTIAN Jean-Pierre, 1997, "Minorités religieuses et confessionalisation de la politique en Amérique latine "Archives des Sciences Sociales des Religions, 97, pp. 97-114.

-, 2007, "Quand la pluralité religieuse change la donne politique en Amérique latine » Esprit, mars-avril, pp. 178-195.

Bezerra Marcos Otávio, 1999, Em nome das "bases ». Política, favor e dependência pessoal, Rio de Janeiro, Relume-Dumará.

CAmpos Leonildo Silveira, 2006, " Os políticos de Cristo - uma análise do comportamento político de protestantes históricos e pentecostais no Brasil », in Burity J., Machado M.D.C., (éds.), Os votos de Deus. Evangélicos, política e eleições no Brasil, Rio de Janeiro, Fundação Joaquim Nabuco, Ed. Massangana, pp. 29-89.

-, 2000, "Composições e recomposições do poder espiritual e político ", Tempo e Presença, 22(313), pp. 20-23.

CARneIro Leandro Piquet, 1998, «Cultura Cívica e Participação Política entre Evangélicos », in Fernandes R. C. (éd.), Novo Nascimento. Os evangélicos em casa, na Igreja e na Política, Rio de Janeiro, Ed. Mauad, pp. 81-110.

Carvalho José Murilo de, 1997, « Mandonismo, coronelismo, clientelismo: uma discussão conceitual », Dados, 40-2, pp. 102-123.

CoRTen André, 1997, "Pentecôtisme et politique en Amérique Latine », Problèmes d'Amérique latine, 24, pp. 11-32.

-, 1999, «Pentecôtisme et néo-pentecôtisme au Brésil », Archives de Sciences Sociales des Religions, 105, pp. 163-183.

Diniz Eli, 1982, Voto e Máquina Política. Patronagem e Clientelismo no Rio de Janeiro, Rio de Janeiro, Paz e Terra.

23. Voir, à ce sujet, le numéro thématique d'Archives de Sciences Sociales des Religions: "Les laïcités dans les Amériques », 146, 2009. 
Fernandes Rubem Cesar, 1996, Novo Nascimento. Os evangélicos em casa, na Igreja e na Política, Rio de Janeiro, Ed. Mauad.

FonseCA Alexandre Brasil, sous presse, Religion and Democracy in Brazil (1998-2001): a study of the leading evangélical politicals.

FRESTON Paul, 1994, "Breve história do pentecostalismo brasileiro ", in Valle R., Sarti I., (éds.), Nem anjos nem demônios. Interpretações sociológicas do Pentecostalismo, Petrópolis, Editora Vozes, pp. 67-162.

-, 1997, «Protestantismo e Democracia no Brasil », Lusotopie, pp. 329-340, www. lusotopie.sciencespobordeaux.fr/freston.rtf

GAUCHET Marcel, 2003, «Neutralité, pluralisme, identités : les religions dans l'espace démocratique ", in Ferrenczi T. (éd.), Religion et politique, une liaison dangereuse? Paris, Éditions Complexe, pp. 55-60.

Giumbelli Emerson, 2002, O fim da religião. Dilemas da liberdade religiosa no Brasil e na França, São Paulo, Attar Editorial, CNPq/PRONEX.

Kaufman Robert, 1977, "Corporatism, Clientelism, and Partisan Conflict: a Study of Seven Latin American Countries ", in Malloy J.M., (éd.), Authoritarianism and Corporatism in Latin America, Pittsburgh, University of Pittsburgh Press.

Hervieu-Léger Danièle, 1999, Le pèlerin et le converti, Paris, Flammarion.

KusChNir Karina, 1996, "Cultura e representação política no Rio de Janeiro ", in Goldman M., Palmeira M., (éds.), Antropologia, voto e representações política, Rio de Janeiro, Contra Capa Livraria, pp. 183-202.

LÖwy Michel, 1997, "L’Église en Amérique latine : le cas brésilien ", in Michel Patrick (éd.), Religion et Démocratie. Paris, Albin Michel, pp. 198-209.

Machado Maria das Dores Campos, 2006, A participação dos evangélicos nas eleições, Rio de Janeiro, FGV Editora.

Mariano Ricardo, 2001, Análise sociológica do crescimento pentecostal no Brasil, São Paulo, Thèse de Doctorat de Sociologie, Université de São Paulo.

Mariano Ricardo, Hoff Marcio, Dantas Toty Ypiranga, 2006, «Evangélicos sanguessugas, presidenciáveis e candidatos gaúchos: a disputa pelo voto dos grupos religiosos ", Debates do NER, Porto Alegre, 7-10, pp. 65-78.

MARY André, 2002, «Le pentecôtisme brésilien en terre africaine. L'universel abstrait du Royaume de Dieu " Cahiers d'études africaines, 167, pp. 463-478.

Oro Ari Pedro, 1996, Avanço pentecostal e reação católica, Petrópolis, Ed. Vozes.

-, 2003, «A política da Igreja Universal e seus reflexos nos campos religioso e político brasileiros », Revista Brasileira de Ciências Sociais, São Paulo, 18-53, pp. 53-69.

Pace Enzo, 1998, "O futuro das religiões na Europa ", Religião e Sociedade, V-19, pp. 19-28.

PEDDE Valdir, 2005, "Cabeça, sim; cauda, não : um estudo antropológico sobre os evangélicos na Assembléia legislativa do Estado do Rio Grande do Sul », Thèse de doctorat en antropologie, PPGAS/UFRGS, Porto Alegre.

Ribeiro Renato Janine, 2002, "Religião e Política no Brasil contemporâneo ", in Friedman L. C. (éd.), Política e Cultura Século, XXI, Rio de Janeiro, ALERJ, Relumé Dumara, pp. 99-110.

SAnchis Pierre, 1994, "O Repto Pentecostal à Cultura Católico-Brasileira ", in Valle R., Sarti I., (éds.), Nem Anjos Nem Demônios, Petrópolis, Vozes, pp. 34-63. 
Santos Marcio Martins dos, 2005, "Tribunos do povo, servos de Deus ": um estudo antropológico sobre políticos e religião na cidade de Porto Alegre, Thèse de doctorat en anthropologie, PPGAS/UFRGS, Porto Alegre.

SEman Pablo, 2000, A «fragmentação do cosmos »: um estudo sobre as sensibilidades de fiéis pentecostais e católicos de um bairro da Grande Buenos Aires, Thèse de doctorat, PPGAS/UFRGS, Porto Alegre.

Willaime Jean-Paul, 1999, " Le pentecôtisme : contours et paradoxes d'un protestantisme émotionnel ", Archives de Sciences Sociales des Religions, 105, pp. 5-28.

\section{Résumé}

L'article tente de faire un bilan de vingt ans de présence évangélique dans la politique institutionnelle brésilienne, surtout à la Chambre des Députés à Brasilia. Il en ressort que la motivation la plus importante de l'engagement pentecôtiste dans la politique, à savoir l'intention d'injecter une forte charge morale dans les affaires politiques, devient, paradoxalement, de nos jours la raison principale de l'éloignement des fidèles de la politique. Ce désenchantement est dîn non seulement au fait que, dans la perspective de ce groupement religieux, la politique en général est "une chose du Diable » mais, plus spécialement, parce que certains députés évangéliques, élus comme " hommes de Dieu ", sont impliqués dans des actions illicites et des pratiques de corruption.

Mots-clés : Brésil, pentecôtisme, politique.

\section{Abstract}

This article presents an overview of the past twenty years regarding the presence of evangelicals in Brazilian institutional politics, especially in the Chamber of Deputies in Brasilia. We argue that the major motivation for the Pentecostal involvement in politics-i.e., the moralization of politics-has, today, paradoxically become the major reason for Pentecostals' estrangement from politics. This estrangement is due to the fact that, according to the perspective of this religious segment, not only politics in general but, in particular, certain evangelical deputies have been implicated in illicit actions and in corrupt practices.

Key words: Brazil, Pentecostalism, politics.

\section{Resumen}

El articolo efectúa una evaluación de veinte años de presencia evangélica en la política institucional brasilera, especialmente en la Cámara de Diputados en Brasilia. Propone que la motivación original de la inserción pentecostal en la politica-el inyectarle una fuerte carga moral- se vuelve, paradojalmente, la razón más importante de su actual distanciamiento de la misma. Este alejamiento se debe al hecho de que, de acuerdo con la perspectiva pentecostal, no solamente la politica en general sino también ciertos diputados evangélicos están implicados en acciones ilícitas y en prácticas de corrupción.

Palabras clave: Brasil, pentecostalismo, politica. 